\title{
Structure of the RECK CC domain, an evolutionary anomaly
}

\author{
T Chang ${ }^{1,2}$, F Hsieh ${ }^{2}$, P Smallwood ${ }^{2}$, S Gabelli ${ }^{3}$, J Nathans ${ }^{1,2}$ \\ ${ }^{1}$ Department of Molecular Biology and Genetics, Johns Hopkins University School of Medicine, \\ Baltimore, USA, ${ }^{2}$ Howard Hughes Medical Institute, Johns Hopkins University School of Medicine, \\ Baltimore, USA, ${ }^{3}$ Department of Biophysics and Biophysical Chemistry, Johns Hopkins University \\ School of Medicine, Baltimore, USA \\ tchang37@jhmi.edu
}

Five small and homologous protein domains (the CC-domains) at the N-terminus of the RECK (Reversioninducing Cysteine-rich Protein with Kazal Motifs), a multi-domain glycosyl-phosphatidyl-inositol (GPI)-anchored protein, play essential roles in signaling by WNT7A and WNT7B in the context of central nervous system angiogenesis and blood-brain barrier formation and maintenance (1-3). Here, we have developed an integrated strategy to determine the crystal structure of CC domain 4 (CC4) and find that it folds into a compact 4-helix bundle with three disulfide bonds (4). The CC4 structure, together with homology modeling of CC1, reveals the surface locations of critical residues that were shown in previous mutagenesis studies (5-8) to mediate G-protein coupled receptor (GPR)124 binding and WNT7A/WNT7B recognition and signaling. Surprisingly, sequence and structural homology searches reveal no other cell-surface or secreted domains in vertebrates that resemble the CC domain, a pattern that is in striking contrast to other ancient and similarly sized domains, such as Epidermal Growth Factor, Fibronectin Type 3, Immunoglobulin, and Thrombospondin type 1 domains, which are collectively present in hundreds of proteins. References: 1. J. Oh et al., The membrane-anchored MMP inhibitor RECK is a key regulator of extracellular matrix integrity and angiogenesis. Cell 107, 789-800 (2001). 2. T. Muraguchi et al., RECK modulates Notch signaling during cortical neurogenesis by regulating ADAM10 activity. Nat Neurosci 10, $838-845$ (2007). 3. B. Vanhollebeke et al., Tip cell-specific requirement for an atypical Gpr124- and Reckdependent Wnt/beta-catenin pathway during brain angiogenesis. Elife 4, (2015). 4. TH. Chang, FL. Hsieh, P. M. Smallwood, S. G. Gabelli, J. Nathans, Structure of the RECK CC domain, an evolutionary anomaly. Proc Natl Acad Sci U S A, (2020). In press 5. C. Cho, P. M. Smallwood, J. Nathans, Reck and Gpr124 Are Essential Receptor Cofactors for Wnt7a/Wnt7b-Specific Signaling in Mammalian CNS Angiogenesis and Blood-Brain Barrier Regulation. Neuron 95, 1056-1073 e1055 (2017). 6. M. Eubelen et al., A molecular mechanism for Wnt ligand-specific signaling. Science 361, (2018). 7. M. Vallon et al., A RECK-WNT7 Receptor-Ligand Interaction Enables Isoform-Specific Regulation of Wnt Bioavailability. Cell Rep 25, 339-349 e339 (2018). 8. C. Cho, Y. Wang, P. M. Smallwood, J. Williams, J. Nathans, Molecular determinants in Frizzled, Reck, and Wnt7a for ligandspecific signaling in neurovascular development. Elife 8, (2019).

Acta Cryst. (2020). A76, a49 